\title{
Advances in the curative management of oesophageal cancer
}

\author{
Jarlath C. Bolger $\mathbb{D}^{1}{ }^{1}$, Claire L. Donohoe ${ }^{1}$, Maeve Lowery ${ }^{1}$ and John V. Reynolds $\mathbb{D}^{1 凶}$
}

(c) The Author(s) 2021

The incidence of oesophageal cancer, in particular adenocarcinoma, has markedly increased over the last four decades with adenocarcinoma becoming the dominant subtype in the West, and mortality rates are high. Nevertheless, overall survival of patients with oesophageal cancer has doubled in the past 20 years, with earlier diagnosis and improved treatments benefiting those patients who can be treated with curative intent. Advances in endotherapy, surgical approaches, and multimodal and other combination therapies have been reported. New vistas have emerged in targeted therapies and immunotherapy, informed by new knowledge in genomics and molecular biology, which present opportunities for personalised cancer therapy and novel clinical trials. This review focuses exclusively on the curative intent treatment pathway, and highlights emerging advances.

British Journal of Cancer (2022) 126:706-717; https://doi.org/10.1038/s41416-021-01485-9

\section{INTRODUCTION}

Oesophageal cancer, comprising mainly oesophageal adenocarcinoma (OAC) and squamous cell carcinoma (SCC), is the seventh most common cancer worldwide, and is responsible for $\sim 450,000$ deaths per year [1]. While historically viewed as a cancer with a dismal prognosis, encouraging trends have emerged across several domains. First, reports of the International Cancer Benchmarking Partnership (ICBP-SURVMARK-2 project), which compare the 1995-1999 to 2011-2014 time periods, highlight an approximate doubling of 5-year survival for both OAC and SCC across seven high-income countries, with the greatest impact in patients under 75 years of age $[2,3]$. Second, in an era in which combination therapies rather than surgery alone are standard in the curative approach to patients presenting with locally advanced disease, a modern benchmark for 5-year survival approaches $50 \%$, which is also an approximate doubling over a 20 year time period [4-6]. Third, an increase in early detection rates of mucosal and submucosal lesions through a combination of greater cancer awareness, surveillance of Barrett's oesophagus (a precursor to oesophageal cancer), management of gastrooesophageal reflux disease (GORD; a risk factor for Barrett's oesophagus and, hence, for oesophageal cancer) and advances in staging has permitted the relatively low-risk treatment of selected patients with endotherapy approaches (endoscopic eradication therapy, EET), such as endoscopic mucosal resection (EMR), endoscopic submucosal dissection (ESD) and radiofrequency ablation (RFA) (Box 1) [7-9].

Furthermore, advances in genomics and molecular research, and the advent of immunotherapeutic and targeted approaches, have improved our understanding of oesophageal carcinogenesis and tumour biology and enabled the development of novel approaches that could improve outcomes [10-13]. Finally, advances in surgery, including standardisation of the extent of resection and lymphadenectomy, and improvements in perioperative care, and in the array of approaches, including minimally invasive and robotic-assisted techniques, have presented opportunities to improve both oncological and operative outcomes [14-16].

Despite these clinical and scientific advances, however, several concerns exist in everyday multidisciplinary team discussions and decision-making regarding patients who can be treated with curative intent. These concerns include the criteria for endotherapy, the choice of neoadjuvant therapy, differential considerations for SCC and OAC, the consideration of a non-operative surveillance approach in patients with an apparent complete clinical response to neoadjuvant therapy, the approach to surgery, and the role of immunotherapy and targeted therapy. This review focuses exclusively on these themes in the curative intent treatment pathway, and discusses existing standards, emerging advances and key controversies. The authors acknowledge significant developments in staging, assessment of treatment response, perioperative care, targeted radiation and proton therapy, surgery for oligometastatic disease and diagnostics, and refer the readers to relevant reviews on these topics $[17,18]$ however, owing to word limitations and our clinical and academic focus, we have structured and prioritised this review into five major sections.

\section{ENDOTHERAPY FOR MUCOSAL AND SUBMUCOSAL CANCER}

Figure 1 gives an overview of staging of early tumours as regards their depth of invasion.

The incidence of OAC has increased in parallel with the rising incidences of GORD and Barrett's oesophagus in Western

\footnotetext{
${ }^{1}$ Trinity St. James's Cancer Institute, Dublin 8, Ireland. ${ }^{凶}$ email: reynoljv@tcd.ie
} 
Box 1:. Endotherapies currently in widespread use in oesophageal cancer

\begin{tabular}{ll} 
Endotherapy & Use \\
\hline $\begin{array}{l}\text { Radiofrequency } \\
\text { ablation }\end{array}$ & $\begin{array}{l}\text { Medium frequency alternating current is used to } \\
\text { ablate Barrett's mucosa with low- or high-grade } \\
\text { dysplasia }\end{array}$ \\
\hline
\end{tabular}

Endoscopic mucosal resection

Areas of mucosa are sucked into endoscope cap, snared, resected and sent for histological analysis

Endoscopic submucosal dissection

Chromoendoscopy then resected en-bloc and sent for histological analysis

Use of staining agents such as acetic acid or Lugol's iodine to show abnormal areas of mucosa
Lesions are raised with submucosal injection

\section{Benefits}

Potential for eradication of Barrett's Prevent progression to invasive malignancy

Prevent morbidity and mortality previously associated with oesophagectomy for dysplasia

May allow complete resection of early cancers (T1a m1-3 and sm1) May act as a staging procedure May allow resection of nodules and then progression to RFA

Allows en-bloc resection of lesions Allows more accurate assessment of margins and potential invasion

\section{Drawbacks}

Requirement for multiple sessions

Potential for stricture

formation

Potential to miss

submucosal lesions

Complications include bleeding and perforation Potential for stricture

May need multiple EMRs Needs close surveillance Does not estimate nodal burden

Technically challenging Higher risk of bleeding and perforation compared with EMR

Allows detection and monitoring of early Inter-observer variability mucosal lesions

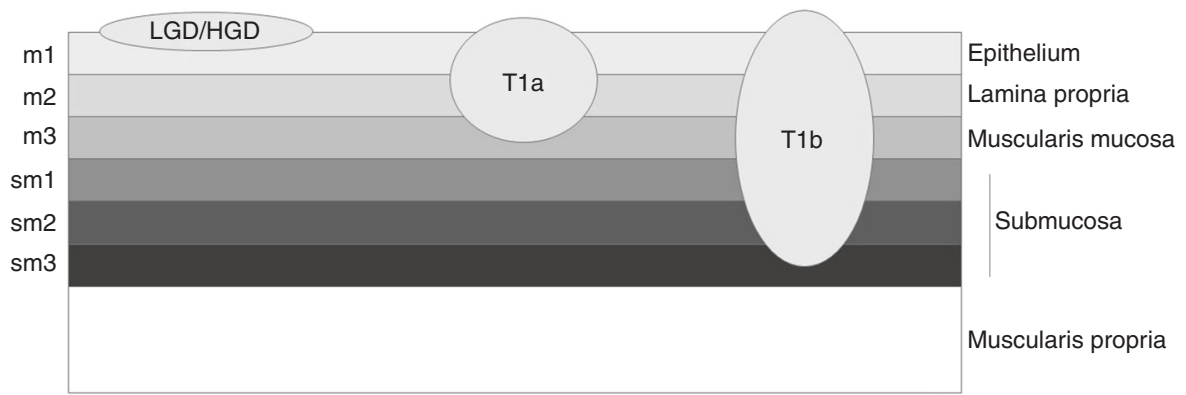

Fig. 1 Overview of the staging of early oesophageal cancers based on their depth of invasion. Low-grade and high-grade dysplasia are confined to the epithelium. T1a tumours are separated based on their depth of invasion (m1-3). T1b tumours invade into the submucosal layer and are further subdivided based on depth of invasion (sm1-3).

populations. As access to endoscopy improves and hence a lower threshold for endoscopy and technological improvements have resulted in an increase in the detection of dysplastic lesions and early cancers $[19,20]$. Furthermore, in the East, the high incidence of SCC of the oral cavity, pharynx and oesophagus, together with strict surveillance policies, is conducive to the diagnosis of early lesions. The assessment and management of apparent mucosal and submucosal disease is enhanced by chromoendoscopy using acetic acid or Lugol's iodine, virtual chromoendoscopy and targeted biopsy protocols [20].

Whereas an oesophagectomy was once the standard approach for patients with high-grade dysplasia (HGD) or mucosal invasion (T1a), the rarity of lymph node metastasis $(<2 \%)$ in this context supports a more targeted local approach. Accordingly, in the latest American Gastroenterological Association guidelines, EET, in the form of EMR and ESD, is preferred to continuing surveillance or oesophagectomy $[8,9]$. The current treatment algorithm reserves oesophagectomy for patients with $\mathrm{T} 1 \mathrm{~b}$ classification, denoting submucosal invasion, in which lymph node metastases occur in approximately $20 \%$ of cases, and for multifocal carcinoma or for lesions that are not amenable to endoscopic resection [9, 19]. Thus, the ability to easily differentiate mucosal (T1a) from submucosal (T1b) invasion would be of value. However, both endoscopic ultrasonography and CT-PET are limited by low sensitivity $[17,21]$.

\section{ENDOSCOPIC FEATURES}

Endoscopic features help to guide the selection of patients for EMR and ESD. The Paris classification (Fig. 2) was developed from previous Japanese classifications, and describes lesions as protruding, excavated or flat (neither protruding nor excavated). It includes type $0-\mathrm{I}$, which denotes elevated or polypoid forms (pedunculated (Ip) and sessile or broad-based (Is)); type 0-II, flat or superficial (Ila flat and elevated, Ilb completely flat, and IIc superficially depressed); and type 0 -III, indicating excavated or ulcerated [22, 23]. Type III lesions and, to some degree, type IIc, might be associated with more aggressive tumour growth, and ulcerated lesions usually reflect deeper level disease that is less suitable for endoscopic therapy for technical reasons as well as being a proxy of understaging.

\section{EET FOR OAC}

When endoscopic resection is considered technically feasible, EMR has become the standard of care for OAC (T1a-m1-3-sm1, early tumours confined to the mucosa or most superficial third of the submucosa) in the West, with ESD preferred in Japan-at minimum to establish a $T$ classification, but in many cases as definitive therapy $[8,9]$. ESD is increasingly utilised for larger suspicious lesions where en bloc EMR is not possible [23, 24]. Modern criteria for curative resection include negative lateral and 


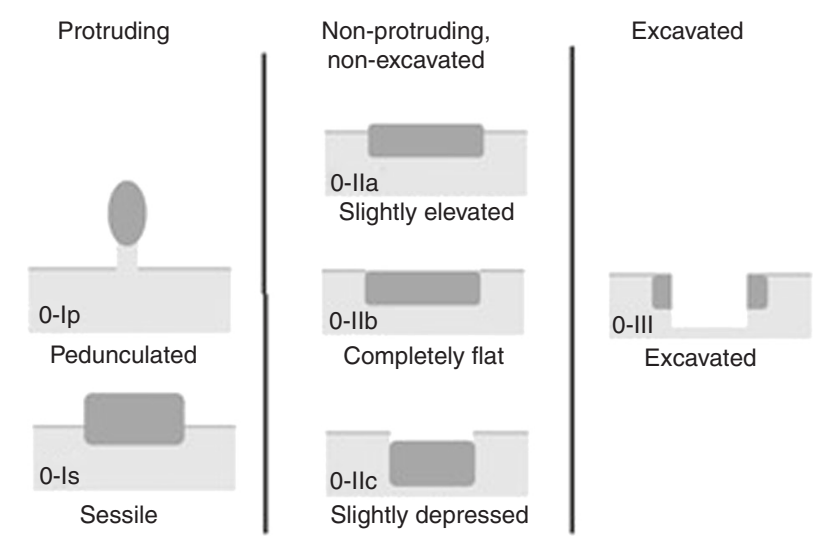

Fig. 2 The Paris endoscopic classification system describes the lesions as protruding, excavated or flat (non-protruding, nonexcavated). This system has not been validated as a prognostic tool in Barrett's oesophagus but studies suggest that sessile and depressed lesions are more likely to contain invasive cancer, with Ila and Ilb lesions at higher risk of associated invasive malignancy.

deep margins (R0), absent lymphatic or vascular invasion (LVI), G1 or G2 grade, well or moderately well differentiated, and absent penetration beyond the first (SM1) layer of the submucosa, approximating to $<500 \mu \mathrm{m}$ depth. Endoscopic resection should be followed approximately 8 weeks later by biopsies of the tumour bed as well as mapping biopsies (mucosal biopsy samples taken sequentially from proximal to distal) of the entire at-risk segment, from $10 \mathrm{~mm}$ above the squamo-columnar junction to $5-10 \mathrm{~mm}$ distal to the $Z$ line of the oesophagus. RFA is commonly used in combination with endoscopic surgery, although photodynamic therapy, argon beam coagulation or cryoablation might also ablate surrounding at-risk epithelium [7]. With such interventions, the recurrence rate is reported to be between $4.5 \%$ and $14.5 \%$, with a median time to recurrence of approximately 2 years, which supports regular endoscopic surveillance typically at 3, 6 and 12 months.

\section{EET FOR SCC}

Endoscopic therapy is less evolved for SCC. The Japanese Esophageal Society recommends the use of vessel irregularities, such as the loss of loop formation and the presence of dilated and tortuous vessels, as a guide to predict the depth of invasion [25]. When combined with conventional endoscopy, magnifying blue laser imaging has been shown to be superior for determining the depth of invasion; moreover, it has low inter-observer variability [26]. Endoscopic surgery for SCC is best studied in Japan-consequently, most of the literature relates to ESD rather than EMR. Early lesions can be resected, assuming that they are less than $5 \mathrm{~cm}$ in axial dimensions and not completely circumferential, as can all lesions with B1 vessel pattern (predicted infiltration T1a superficial to muscularis mucosa), and a B2 vessel pattern, suggesting extension to muscularis mucosa or the superficial submucosa (SM1) $[25,26]$. R0, G1/G2 grade, and absent LVI are good prognostic indicators, and superficial infiltration of the submucosa $(\leq 200 \mu \mathrm{m})$ is acceptable in selected cases $[27,28]$. An involved margin and adverse pathology such as LVI indicate the need for additional treatment-either an oesophagectomy or adjuvant chemoradiotherapy. A series of 176 patients who initially had ESD, of whom 87 had pT1a with LVI and the remainder had pT1b tumours, and who received adjuvant chemoradiotherapy, reported an excellent 3-year survival of $90 \%$, thus presenting this approach as a valid alternative to oesophagectomy [29].
OPTIMUM APPROACH TO LOCALLY ADVANCED OAC AND SCC The seminal randomised clinical trial (RCT) examining the use of neoadjuvant therapy in oesophageal cancer, a modern benchmark for subsequent trials in OAC and SCC, is the CROSS Trial, a multicentre Dutch study of 366 patients, $75 \%$ of whom had OAC, which recruited from 2004 to 2008, and published initially in 2012 [5]. This RCT conclusively established that neoadjuvant chemoradiotherapy (paclitaxel, carboplatin and $41.4 \mathrm{~Gy} / 23$ fractions) prior to resection was superior to surgery alone in patients with locally advanced cancer. Median survival was 45 months for OAC and 81.6 months for SCC, compared with 24 months in the surgery-only group. The overall 5 -year survival was $47 \%$ with multimodal therapy (neoadjuvant therapy followed by definitive surgical resection), and no evidence of added operative mortality or major morbidity was evident. In spite of the outstanding outcomes, key concerns persist-in particular, whether this approach is superior to perioperative chemotherapy without radiation, and whether definitive chemoradiotherapy is a valid alternative to perioperative chemotherapy. Due to differences in tumour biology, which are particularly relevant in terms of the response to radiation therapy, each pathological type of oesophageal cancer will be discussed separately.

\section{OAC}

In some parts of the World-particularly the UK, France and Germany-chemotherapy prior to and after surgery was the dominant approach prior to the CROSS trial, and subsequently has been slow to change.

\section{Challenging the CROSS trial}

This reluctance to change is, to a large extent, due to level I evidence (in the UK and France) from RCTs of gastric adenocarcinoma and OAC predating CROSS which clearly established the superiority of perioperative chemotherapy compared with surgery alone [30, 31]. In addition, some reports of poor operative outcomes following preoperative radiation therapy at that time highlighted safety concerns about potential added operative risk [32]. The MAGIC RCT, designed for gastric and gastrooesophageal junction tumours, and latterly including oesophageal tumours, arguably had the greatest impact in changing treatment approaches. Three cycles of epirubicin, cisplatin and fluorouracil before and after surgery resulted in a 5 -year survival of $36 \%$ in the treated arm compared with $23 \%$ for surgery alone [30]. A similar finding was observed in the contemporaneous French Accord trial, in which $75 \%$ of patients had junctional or oesophageal adenocarcinoma, with a 5-year survival of $38 \%$ in patients receiving perioperative therapy compared with $24 \%$ in those undergoing surgery alone [31]. In the UK Medical Research Council OE05 RCT, published in 2017, 897 patients from 78 UK centres were randomised to receive either two cycles of cisplatin and fluorouracil or four cycles of epirubicin, cisplatin and capecitabine preoperatively, with surgery standardised to a two-stage radical en bloc resection. Median survival was 23.4 (95\% Cl, 20.6-26.3) and 26.1 (22.5-29.7) months, respectively, thus providing no added rationale for triplet therapy [33].

In this overall context, results from the German FLOT-4 RCT, published in 2019, look set to be a game changer globally and have already challenged existing patterns of care [4]. In this Phase II/III RCT, 716 patients with gastric, lower oesophageal or gastrooesophageal junction adenocarcinoma received either a modified MAGIC protocol or the FLOT regimen of fluorouracil plus leucovorin, oxaliplatin and docetaxel administered as four preoperative and four post-operative cycles. The median survival of 50 months versus 35 months in favour of FLOT was particularly impressive, and a pathological complete response ( $p C R$ ) rate of $16 \%$ compared with $6 \%$ for the control (modified MAGIC protocol) 
group approached published $\mathrm{pCR}$ outcomes using neoadjuvant chemoradiation [6]. The sole, but major, caveat was a high incidence of grade 3 or 4 neutropenia, at $51 \%$, and severe infections, at $18 \%$, and just $46 \%$ of patients completed all cycles of chemotherapy. A case series $(n=131)$ in patients with OAC also reported impressive outcomes in response to FLOT, with a major pathological response rate (MPR) of 31\%, a 5-year survival of $51 \%$, and a $35 \%$ incidence of recurrence (with just $16 \%$ being locoregional recurrence) [34].

\section{Multimodal therapy versus chemotherapy}

Accordingly, whether neoadjuvant chemoradiotherapy, best represented by the CROSS regimen, is superior to optimum perioperative chemotherapy, with FLOT increasingly being seen as the standard, is of major current interest. Previous studies, including the Phase III POET RCT, which closed early, a Phase II RCT (NeoRES-1), and a small Australasian RCT, failed to show any survival advantage using multimodal protocols compared with perioperative chemotherapy [35-37] (Table 1). In NeoRES-1, the 3 -year survival was $47 \%$ versus $49 \%, P=0.77$, in multimodal versus chemotherapy alone, respectively, despite higher $\mathrm{pCR}$ rates ( $28 \%$ versus $9 \%$ ) and lower numbers of pathologically (ypNo) involved nodes [36] (38\% vs $65 \%, P=0.001)$. An important further caveat was the occurrence of increased perioperative mortality rates, at $6 \%$ vs $3 \%$ for NeoRES and $10.2 \%$ vs $3.8 \%$ for POET for multimodal versus chemotherapy alone,' respectively [36, 37].

Several major, well-designed, Phase III RCTs are currently active and will address whether a multimodal approach is superior to chemotherapy alone (Fig. 3). ESOPEC is a multicentre German RCT $(n=428)$ directly comparing FLOT with CROSS for OAC, with a power calculation based on FLOT being 13\% superior to CROSS [38]. Neo-AEGIS $(n=540)$ is a European RCT that initially used a modified MAGIC regimen (EOX) as the control arm versus CROSS, but since 2018 has permitted the use of FLOT [39]. An initial power calculation for $10 \%$ superiority to CROSSwas adjusted for non-inferiority after the initial futility analysis. TOPGEAR $(n=$ 570) compares perioperative chemotherapy (modified MAGIC but FLOT permitted since 2017) with neoadjuvant chemoradiotherapy (45 Gy and cisplatin/fluorouracil (capecitabine, a pro-drug of 5-FU) in gastric and oesophageal adenocarcinoma, and is powered on superiority of the multimodal arm [40]. These trials include over 1000 randomised patients and will provide considerable novel data, and should determine whether there is a superior arm and new gold standard, or whether the approaches are equivalent. Important secondary endpoints include recurrence patterns, postoperative morbidity and mortality rates, and treatmentrelated toxicity. Irrespective of the overall conclusions, the limitations of these RCTs will be specific clinical scenarios that might be excluded, such as CT4a, as well as the optimum management of cN3, ypN1-3 post neoadjuvant chemoradiotherapy, and signet ring cell carcinoma/diffuse pathological subtypes as distinct from the more common intestinal pathological subtype. Another RCT on this theme, RACE, has commenced in Germany; it is designed as a prospective, randomised, stratified Phase III clinical trial comparing standard FLOT with FLOT plus preoperative radiation therapy using a $45 \mathrm{~Gy}$ dose [41]. This RCT aims to recruit 340 patients, with progression-free survival as the primary endpoint.

\section{Optimum radiation therapy plus chemotherapy}

Another multimodal strategy is to explore optimum combinations of radiation therapy with chemotherapy in the neoadjuvant setting. NEOSCOPE randomised 85 patients to receive either neoadjuvant oxaliplatin-capecitabine or carboplatin-paclitaxel with $45 \mathrm{~Gy}$ concurrent radiation therapy, and reported a higher pCR rate in the carboplatin-paclitaxel group [42]. The PROTECT-1402 compares FOLFOX plus radiation with the CROSS regimen [43]. The aim is to recruit 106 patients, with the primary outcome being 

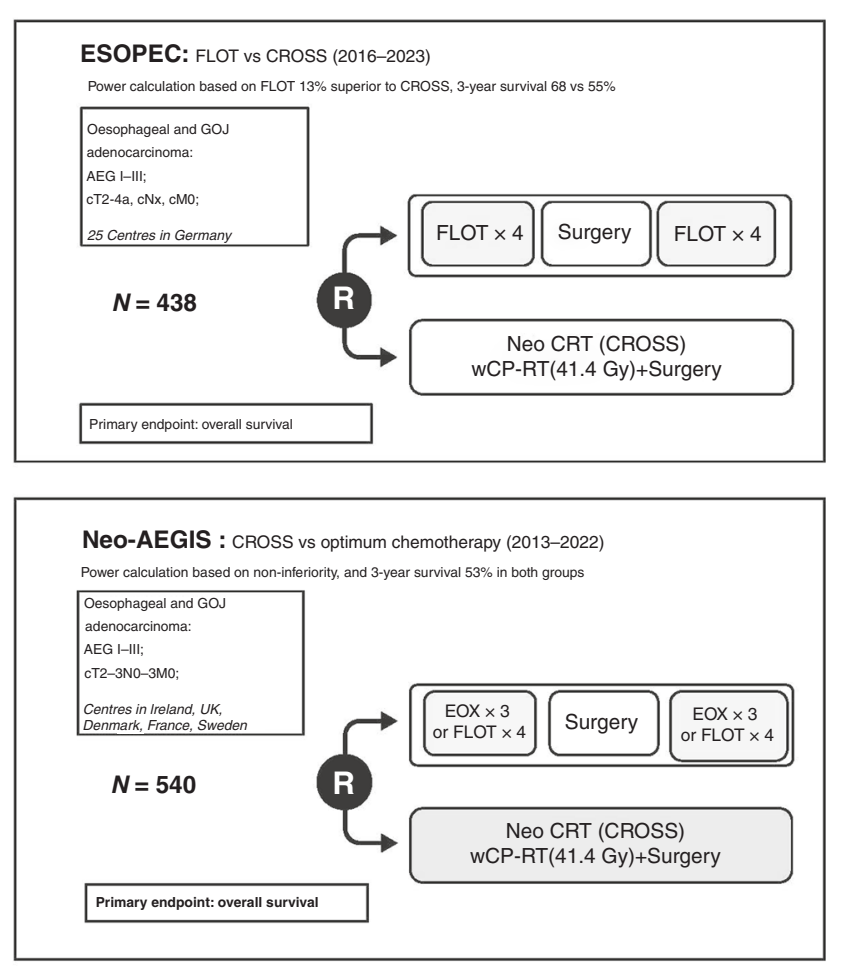

TOPGEAR: (Multimadal vs optimum chemotherapy : 2009-2023)

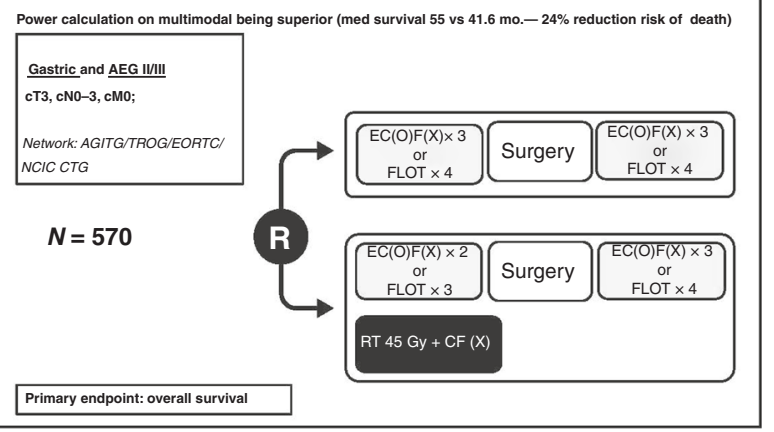

Fig. 3 Key ongoing trials of perioperative and neoadjuvant therapy for oesophageal adenocarcinoma. FLOT fluorouracil, leucovorin, oxaliplatin and docetaxel, CROSS paclitaxel, carboplatin and $41.4 \mathrm{~Gy} / 23$ fractions, EOX epirubicin, oxaliplatin, capecitabine, $\mathrm{EC}(\mathrm{O}) \mathrm{F}(\mathrm{X})$ epirubicin, cisplatin (or oxaliplatin), fluorouracil (or capecitabine).

R0 resection rates. In the Alliance Trial, patients $(n=257)$ were randomised to a modified FOLFOX or carboplatin-paclitaxel combined with radiotherapy $(50.4 \mathrm{~Gy} / 28$ fractions) [44]. Interestingly, if a patient was deemed to be a non-responder by a second CT-PET [ $<35 \%$ decrease in standardised uptake value (SUVmax)], he/she crossed over to the other chemotherapy regimen. The 2-year overall survival was $61.8 \%$ (95\% Cl 55.7-68.5\%), with a median of 40.2 months for responders and 27.4 months for nonresponders, and the best outcomes in responders were with the FOLFOX combination therapy. This RCT also highlighted the positive predictive impact of a CT-PET-determined metabolic response on prognosis and how it might be adapted to a trial design, as reported originally in the MUNICON trials [45-47].

\section{SCC}

Although most trials on oesophageal cancer have included OAC and SCC patients together, the $\mathrm{PCR}$ rates after neoadjuvant chemoradiotherapy of $49 \%$ versus $23 \%$ for SCC versus adenocarcinoma, respectively, in the CROSS trial illustrate the higher sensitivity of SCC to radiation regimens $[5,6]$. Although the number of treated SCC patients was small $(n=41)$, the impressive response rate and survival has established a modern benchmark. In a national, high volume centre, multimodal therapy was associated with a 5-year disease-specific survival of $62 \%$ in a series of 75 patients with SCC [48]. An international RCT (NEOCRTE5010) of 451 patients with SCC showed a median survival of 100 months versus 66.5 months in favour of multimodal therapy, with a pCR rate of $43.2 \%(P=0.025)$ [49].

\section{Differing treatment approaches}

However, despite the apparent success of multimodal therapy for SCC patients, there are contrary views on this topic, and practice varies widely. The East has seen a gradual shift in the SCC treatment algorithm from adjuvant therapy to neoadjuvant approaches, with a preference largely for chemotherapy alone, and with an increasing use of docetaxel, a key component of the FLOT regimen. Reported response rates are over $60 \%[50,51]$. Currently, an RCT (JCOG1109, NExT study) conducted by the Japan Esophageal Oncology Group has completed recruitment with a view to comparing three neoadjuvant therapy regimens: cisplatin and 5-fluorouracil versus cisplatin, 5-fluorouracil and docetaxel versus cisplatin, 5-fluorouracil and radiation therapy (41.4 Gy) [52]. In the West, conversely, the debate largely relates to multimodal therapy versus definitive chemoradiotherapy, and both are accepted as equivalent within international guidelines and in the Cochrane database of systematic reviews [53]. This judgement of equipoise is largely based on two RCTs. A German trial $(n=189)$ published in 2005 compared chemoradiotherapy (cisplatin, etoposide-40 Gy) followed by surgery with continued chemoradiotherapy increasing to $65 \mathrm{~Gy}$ in patients with cT3 and cT4 tumours, with all patients having had initial induction chemotherapy with fluorouracil, leucovorin, etoposide and cisplatin [54]. Those undergoing surgery demonstrated an improved 2-year progression-free survival (64\% versus $40 \%, P=0.003$ ) compared with those receiving continued CRT, but showed no improvement in overall survival and a high in-hospital mortality rate $(11.3 \%)$. A French RCT (FFCD 9102) similarly showed no difference in survival in patients treated with induction therapy (cisplatin and 5fluorouracil-RT) and randomised, if responding, to either continued chemoradiotherapy or surgery; however, locoregional relapse was higher in the chemoradiotherapy group [55]. Conversely, both RCTs reported a significant increase in treatment-related mortality in the multimodal cohorts $[55,56]$. Accordingly, no level I data have established a standard of care for locally advanced SCC globally. Nevertheless, the impressive outcomes from CROSS and other series of multimodal therapy and, more recently, data analysis of the (US) National Cancer Database of 19,532, which reported a doubling of survival for multimodal compared with definitive chemoradiotherapy in nonrandomised comparisons, provides support for an approach that includes surgery as a standard of modern practice, particularly in the modern era in which the risk of operative mortality is low [56]. In Japan, although preoperative chemotherapy is more standard than multimodal therapy, a JCOG trial (0909) of 50 Gy RT with cisplatin and 5-fluorouracil in SCC Stage II/III patients resulted in excellent outcomes, and this regimen is currently used as standard therapy in patients who do not wish to have surgery or are relatively unfit [57].

\section{Influence of radiation dose}

What of higher doses of radiation in the definitive chemoradiotherapy setting? An established protocol involves $50.4 \mathrm{~Gy}$, particularly in North America, and no clear evidence exists of the benefit of increasing doses [58, 59]. The ARTDECO (61.6 vs $50.4 \mathrm{~Gy}$ ) and CONCORDE/PRODIGE 26 (76 vs $50 \mathrm{~Gy}$ ) trials compare higher 
doses to standard doses of radiation therapy [60,61]. In 2020, ARTDECO reported no improvement $(P=0.08)$ in local progression-free survival with a higher dose of radiation therapy, and an increase in grade $4 / 5$ toxicity [61]. In the UK, SCOPE 2 (NCT02741856) is examining dose escalation from 50 to $60 \mathrm{~Gy}$ in definitive chemoradiotherapy [62].

\section{Alternative approaches}

Because SCC is less common in Western patients, a proposed RCT with surgery as part of the control arm has struggled to recruit sufficient eligible patients [63]. If definitive chemoradiotherapy is preferred based on application of guidelines, centre preference, patient fitness or preference, then some patients who develop recurrence locally might be suitable for so-called salvage oesophagectomy [56]. Encouragingly, in a large population study, there was no significant difference in median survival for those who underwent neoadjuvant chemoradiotherapy plus surgery compared with those with local recurrence undergoing salvage oesophagectomy (36 months versus 35.5 months, $P=0.8$ ) [56]. Perioperative outcomes were also similar, with no difference in perioperative mortality. This compares favourably with prior series reporting mortality rates of up to $25 \%$ in those undergoing salvage procedures [64]. Finally, proton beam therapy (PBT) is emerging as an interesting alternative to standard radiation therapy regimens, although trial data is currently lacking [65]. PBT offers the potential for reducing off-target side effects while maintaining the dose distribution to the primary tumour. Early work suggests is may be efficacious in OAC and SCC.

\section{SURGERY AS NEEDED ('WATCH AND WAIT') FOR COMPLETE CLINICAL RESPONDERS AFTER NEOADJUVANT THERAPY}

Although salvage surgery is used after definitive chemoradiotherapy for isolated local recurrence, or persistent disease, an emerging concept is whether patients with apparent complete response to neoadjuvant therapy prior to planned surgery can be kept under strict surveillance and only operated on if proven locoregional disease is evident in follow-up. The advantages are clear, and include the avoidance of a major operation with an up to $5 \%$ risk of mortality (as well as attendant major morbidity), in addition to organ preservation. This concept might have gained extra importance during the SARS-CoV-2 pandemic, which has led to alterations in standard care pathways and a pragmatic consideration of such an approach [66].

\section{ASSESSING THE 'WATCH AND WAIT' APPROACH}

The 'watch and wait' rationale appears more compelling for patients with SCC, almost $50 \%$ of whom had a pCR in the CROSS Trial, compared with $25 \%$ of patients with OAC [5]. However, evidence is needed to prove that a complete clinical response equates to a pCR and, to this end, the PreSANO (Surgery as Needed for Oesophageal Cancer) study was designed [67]. PreSANO was a single arm, multicentre study from six centres in the Netherlands, and included patients with OAC and SCC. In the design, patients with an apparent complete clinical response based on endoscopic assessment, bite-on-bite (deep) biopsies and endoscopic ultrasonography at 6 weeks had a further clinical response evaluation at 12 weeks, which included CT-PET, endoscopic ultrasonography plus fine needle aspiration of suspicious nodes, and additional biopsies. The study reported good sensitivity (90\%) for detecting residual disease $(>10 \%$ vital residual tumour cells) but poor sensitivity for detecting the complete absence of residual disease, with residual disease often microscopic and confined to the submucosa [67]. A presentation Van Der Wilk B, et al. [68] at the 2020 International Society of Diseases of the Esophagus (ISDE) Meeting involving 256 patients from seven studies undergoing a watch and wait approach reported a pooled 5-year survival of $58 \%$, with locoregional recurrence of $33 \%$ at 2 years. The limited sensitivity shown in preSANO and other series for individual elements of clinical response evaluation -in particular CT-PET and endoscopic ultrasonography-presents a notable caveat with respect to pursuing this 'watch and wait' approach outside of RCTs, particularly for OAC [69-71]. The preSANO group has activated the SANO trial, which will have as a primary endpoint non-inferiority of a strict surveillance programme versus surgery, if the 12-week clinical response evaluation shows no residual disease [72]. The French Esostrate-Prodige 32 study is similar in design to SANO, but the randomisation method differs [73]. For SCC alone, in four Asian Centres, a preliminary study preSINO (surgery if needed for oesophageal cancer) is also underway based on preSANO principles; this might progress to an RCT [74].

\section{MULTIMODAL THERAPY VERSUS DEFINITIVE CHEMOTHERAPY AND POTENTIAL SALVAGE SURGERY}

Although patients might accept some risk in avoiding surgery, potential downsides exist, as intensive surveillance brings with it the necessity for frequent hospital attendance for procedures, with inherent anxiety and impact on quality of life, in addition to an anticipated requirement for 'salvage' surgery in approximately one in three patients [75]. The NEoadjuvant chemoradiotherapy for Esophageal squamous cell carcinoma versus Definitive chemoradiotherapy with salvage Surgery as needed (NEEDS trial-EudraCR 2020-000149-15) is an important new international $\mathrm{RCT}$, led by the Karolinska Institute, that has recently commenced recruitment. The trial compares CROSS regimen multimodal therapy with definitive chemoradiotherapy and salvage surgery as needed, and is powered for non-inferiority, with a target to recruit 1200 patients and an estimated study completion date of 2031 [76].

\section{IMMUNOTHERAPY AND TARGETED THERAPIES IN THE NEOADJUVANT AND ADJUVANT CONTEXT}

Advances in cancer genomics, molecular biology and immunology are bringing about a modern revolution in cancer therapy [10-13]. In the context of oesophageal cancer, this revolution is mainly evident in metastatic or advanced incurable disease, where several novel approaches are approved in first, second and third-line protocols.

\section{TARGETED THERAPIES}

Key trials of targeted therapies include TOGA, in which trastuzumab, a monoclonal antibody that targets HER2, in combination with chemotherapy improved outcomes compared with chemotherapy alone in patients with HER2 ${ }^{+}$tumours; and REGARD and RAINBOW, in which the vascular endothelial growth factor (VEGF) receptor inhibitor ramucirumab was beneficial as a single agent or combined with paclitaxel, respectively [77-79]. PETRARCA reported improved disease-free survival and overall survival with the addition of trastuzumab and another HER2targeting monoclonal antibody, pertuzumab, to the FLOT regimen in patients with $\mathrm{HER} 2^{+}$, resectable oesophagogastric cancer in a Phase II RCT, with a remarkable pCR of $35 \%$ for the combination therapy [80].

\section{IMMUNOTHERAPIES}

Immune therapies that target 'immune checkpoints' such as programmed cell death protein 1 (PD-1) and its ligand PD-L1, or cytotoxic lymphocyte antigen 4 (CTLA-4) are currently of great interest [81]. A $10-15 \%$ response rate to immune therapies has been reported in patients with recurrent or metastatic 
oesophageal cancer [82]. Advances in the use of immune checkpoint inhibitors for oesophageal cancer have come from combination studies in the first-line setting. In KEYNOTE-590, a combination of the PD-1 inhibitor pembrolizumab with chemotherapy demonstrated a survival benefit compared with chemotherapy alone in patients with OAC or SCC, particularly in tumours with a combined positive score (the CPS, based on the number of PD-L1 positive cells in relation to the total number of tumour cells) $\geq 10[83,84]$. In CHECKMATE-649, in which patients with gastric cancer and OAC were randomised to receive either chemotherapy or chemotherapy plus nivolumab, an overall survival benefit with the addition of the PD-1 inhibitor was seen, particularly in patients with a CPS $\geq 5$ [85]. Key monotherapy trials include ATTRACTION-3, an international study in patients with advanced SCC, which reported a survival advantage for nivolumab compared with investigators' choice chemotherapy in the secondline setting, while KEYNOTE 181 demonstrated a benefit with pembrolizumab over chemotherapy in the second-line setting for patients with SCC and a CPS $\geq 10$, but not for patients with OAC [86]. Interestingly, a combination of pembrolizumab and trastuzumab with chemotherapy resulted in excellent progression-free survival in $\mathrm{HER}^{+}$patients, and is being evaluated in a randomised Phase III trial [87]. The optimal biomarkers of response to immune checkpoint inhibition have yet to be defined; in addition to PD-L1 overexpression, microsatellite instability (MSI), high mutational burden and defects in DNA mismatch repair might also predict a durable response [81].

\section{TARGETED THERAPIES AND IMMUNOTHERAPY IN THE CURATIVE SETTING}

Do these developments in treatments for metastatic and incurable disease translate into improved outcomes in curative therapy? In the same way that FLOT is a potential game changer for OAC, adjuvant immunotherapy might hold similar future promise for both OAC and SCC. In the CHECKMATE-577 RCT, 794 patients with Stage II/III oesophageal cancer were treated with neoadjuvant chemoradiation; patients who did not achieve a pCR following resection were randomised 2:1 to receive adjuvant nivolumab or placebo [88]. At an interim analysis, median disease-free survival was doubled (22.4 versus 11 months, HR 0.69 [Cl:0.56-0.86) $P=$ 0.0003 ) in patients who received nivolumab. This finding is not only exciting, but scientifically plausible, as radiation therapy induces the expression of PD-L1 and the recruitment of immune cells in the tumour microenvironment $[89,90]$.

CHECKMATE-577 provides a compelling evidence base that supports immunotherapy in the postoperative adjuvant setting after neoadjuvant chemoradiotherapy, and is a significant advance in adjuvant therapy for oesophageal cancer. Whether this approach is effective in the neoadjuvant context is unknown, and whether adjuvant therapy complements the effect of optimum perioperative chemotherapy regimens such as FLOT also requires evaluation. Studies are active using pembrolizumab, durvalamab (an anti PD-L1 mAb), and tremelimumab (an antiCTLA-4 mAb) in this context, as are trials combining chemotherapy regimens, including FLOT with immunotherapy or approved targeted therapies [91]. These combination regimens include FLOT-8/DANTE (NCT03421288), which compares the standard perioperative FLOT regimen plus the PD-L1 inhibitor atezolizumab, and FLOT-7/RAMSES (NCT02661971), which uses the FLOT regimen plus ramucirumab [90].

\section{Neoadjuvant immunotherapy and healing}

Another important issue relates to whether immunotherapy can be given safely in the neoadjuvant setting without increasing complications associated with oesophageal surgery-in particular, wound and anastomotic healing-or the postoperative systemic or organ-specific inflammatory response. Relevant data with respect to approaches that impact on inflammation and healing might come from the UK MRC ST03 trial, which randomised 1063 patients with gastric, oesophageal or gastro-oesophageal junction adenocarcinoma to receive epirubicin, cisplatin and capecitabine alone or in combination with the VEGF inhibitor bevacizumab. This trial reported an equivalent 3-year survival of $50.3 \%(95 \% \mathrm{Cl}$ 45.5-54.9) and $48.1 \%$ (95\% Cl 48.1\% (43.2-52.7), respectively, but, of concern, a more than doubling of anastomotic leaks (24\% versus $10 \%$ ) was seen in patients who underwent an oesophago-gastric anastomosis; consequently, recruitment was closed to this cohort based on this analysis [92]. Currently, with limited data published, there is no evidence that immunotherapy in the neoadjuvant context impacts on key operative outcomes; however, this scenario should be carefully studied in prospective evaluation [93, 94].

\section{ADVANCES IN SURGERY IN A MINIMALLY INVASIVE ERA}

Open surgery has been the mainstay of surgery for generations, with the main issues in the context of oesophageal cancer being the value of transthoracic oesophagectomy (TTO) compared with a transhiatal approach (TH), which avoids the need for a thoracotomy, and also whether TTO is superior to an extended total gastrectomy and mediastinal anastomosis for adenocarcinoma of the cardia (Siewert type II) $[95,96]$. TTO comprises a twostage (Ivor Lewis) oesophagectomy with an intrathoracic anastomosis or a three-stage oesophagectomy with a cervical anastomosis. One of the advantages of TTO over TH is the extent of lymphadenectomy that is possible within the thorax, and en bloc resection along tissue planes on the aorta and tracheobronchial tree. TTO allows more accurate lymph node staging due to the nodal yield, and as dissection from surrounding structures is done under direct vision offers the ability to improve circumferential resection margin rates.

\section{MINIMALLY INVASIVE APPROACHES TO OESOPHAGECTOMY}

Consistent with trends across surgery as a whole, the modern era has seen an increase in minimally invasive and robotic-assisted approaches, with emerging evidence suggesting oncological equivalence compared with open surgery, but perhaps an association with less major morbidity [97]. Although minimally invasive oesophagectomy (MIO) was first reported in the early 1990 s, it is in the last decade that it has developed internationally and begun to supplant open approaches $[98,99]$.

Although logic compels the use of MIO compared to open surgery if it can be safely conducted, RCTs are the gold standard to demonstrate superiority or non-inferiority; however, the evidence base to date is limited, and focuses mainly on the occurrence of postoperative pulmonary complications. The key trials are outlined in Table 2. The Dutch TIME trial compared a completely minimally invasive approach with open surgery in 115 patients [100]. The primary outcome-post-operative microbiologically proven respiratory infection within 14 days-was significantly different, at $9 \%$ in the MIO cohort versus $29 \%$ in the open group $(P=0.05)[14,100]$. The French MIRO trial randomised 207 patients to undergo traditional open TTO or a hybrid MIO approach comprising a laparoscopic abdominal phase combined with an open thoracotomy [15]. The primary outcome of major 30 day post-operative complications was significantly decreased, from 64 to $36 \%(P<0.001)$, using the hybrid approach, mainly relating to decreased major respiratory complications.

However, some issues remain. In the TIME trial, the primary endpoint of proven pneumonia in the open group was higher than anticipated and previously reported [101, 102]. In MIRO, Clavien-Dindo grade II severity complications were defined as 'major', which might not be commonly accepted, as Grade II do not require surgical or radiological intervention; and the results 
were equivalent for both treatment arms with respect to $\geq$ grade III severity complications, suggesting that hybrid oesophagectomy may have a more significant impact on less severe complications [15]. A further caveat comes from a registry study from the Netherlands comparing MIO and open approaches, in which an increase in perioperative complications, reoperation rates and length of stay for patients undergoing MIO was reported [101]. This discordance might reflect a learning curve for those training in $\mathrm{MIO}$, or could relate to low-volume centres performing procedures, but it is clear that further real-world data and rigorous clinical audit are necessary to ensure patient outcomes are not compromised by the introduction of new surgical techniques [103]. With respect to current RCTs, no direct comparison of a hybrid approach and a totally MIO approach is currently being investigated. The ROMIO trial aims to compare open, hybrid and $\mathrm{MIO}$ approaches in over 400 patients, with a primary outcome of patient-reported physical status post operatively [104].

\section{ROBOT-ASSISTED APPROACHES}

Throughout most of surgery, robotic-assisted approaches are undergoing testing and evaluation, while modern trainees are increasingly focused on acquiring robotic skills and accreditation. Advantages of a robotic approach might include articulation of the instruments, minimisation of large movements for the surgeon, and better ergonomics and, arguably, this approach might supplant minimally invasive approaches if the considerable costs can be contained. In oesophageal cancer surgery, a robotic approach was first described by Kernstine et al. [105], and advanced in Europe mainly by Professor Van Hillegersberg and colleagues in Utrecht, the Netherlands, and this field of robotic assisted minimally invasive oesophagectomy (RAMIE) has progressed rapidly in the past decade $[16,105]$. The ROBOT Trial compared robotassisted three-stage oesophagectomy and open three-stage oesophagectomy in a study of 109 patients, with a primary endpoint of post-operative complications [16]. RAMIE was associated with decreased overall complications (59\% versus $80 \%, P=0.02$ ), as well as decreased blood loss, reduced pain and earlier functional recovery, with 5 -year survival equivalent between the two approaches, at $42 \%$ versus $43 \%(P=0.74)$, for RAMIE and open, respectively $[16,106]$.

A caveat of a robotic approach as for $\mathrm{MIO}$, relates to anastomotic leak rates, and highlights the need for rigorous mentoring and accreditation, and oversight in training, in the future. A 2020 international registry study reported leak rates of $33 \%$ for robot-assisted hand-sewn anastomosis, $17 \%$ for circular stapled anastomosis and 15\% for linear stapled anastomosis using RAMIE during a particular procedure [107]. These rates are high, and well above reported rates from high-volume centres with standardised stapled or hand-sewn techniques [101]. To this end, the OesophagoGastric Anastomosis Audit examining oesophago-gastric anastomosis might provide some clarity as to the optimal anastomotic techniques in standard oesophagectomy, MIO and RAMIE [108].

\section{CONCLUSION}

Despite the considerable challenge in curing a cancer that frequently presents at an advanced stage and that might have adverse biological features that promote resistance to standard therapies, significant progress has been made in the curative approach to oesophageal cancer. An increasing reserve of RCTs nearing completion, as well as advances in endotherapy, staging, surgery and high-content scientific analysis, in addition to an improved understanding of genomics and the tumour microenvironment offers a real promise of further discovery and improved cure rates. The model for the curative 
approach to locally advanced oesophageal cancer is likely to change in the not-too-distant future. Information on HER2 status, PD-L1 expression, MSI and overall mutational burden-currently the preserve of metastatic disease algorithms - might be increasingly relevant for the incorporation of targeted therapy within treatment plans, and to help guide the response to therapy $[94,109,110]$. Challenges exist, with few targetable mutations in oesophageal cancer, and a high mutational frequency in highly heterogenous tumours. Cost is also an issue, as is affordable, efficient high-throughput sequencing with a clear clinical application whether in stratifying prognosis or in tailoring therapies. Biomarkers are also needed and, in this context, circulating tumour DNA has shown some promise $[111,112]$. As technology and techniques evolve in EET and surgery, including the potential for increased use of artificial intelligence, there may be significant challenges in the introduction of this technology. In addition to significant cost implications, there must be equitable access to novel techniques to ensure patients benefit in a just way. This review, written mainly from the perspective of academic surgeons and oncologists, although focused on five major themes, highlights key advances in medicine and science, and novel vistas that continue to give cause for optimism.

\section{DATA AVAILABILITY}

Not applicable.

\section{REFERENCES}

1. Arnold M, Abnet CC, Neale RE, Vignat J, Giovannucci EL, McGlynn KA, et al. Global burden of 5 major types of gastrointestinal cancer. Gastroenterology. 2020;159:335-49.

2. Arnold M, Rutherford MJ, Bardot A, Ferlay J, Andersson TM, Myklebust TA, et al. Progress in cancer survival, mortality, and incidence in seven high-income countries 1995-2014 (ICBP SURVMARK-2): a population-based study. Lancet Oncol. 2019;20:1493-505.

3. Morgan E, Soerjomataram I, Gavin AT, Rutherford MJ, Gatenby P, Bardot A, et al. International trends in oesophageal cancer survival by histological subtype between 1995 and 2014. Gut. 2021;70:234-42.

4. Al-Batran SE, Homann N, Pauligk C, Goetze TO, Meiler J, Kasper S, et al. Perioperative chemotherapy with fluorouracil plus leucovorin, oxaliplatin, and docetaxel versus fluorouracil or capecitabine plus cisplatin and epirubicin for locally advanced, resectable gastric or gastro-oesophageal junction adenocarcinoma (FLOT4): a randomised, phase 2/3 trial. Lancet. 2019;393:1948-57.

5. Shapiro J, van Lanschot JJB, Hulshof M, van Hagen $P$, van Berge Henegouwen $\mathrm{MI}$, Wijnhoven $\mathrm{BPL}$, et al. Neoadjuvant chemoradiotherapy plus surgery versus surgery alone for oesophageal or junctional cancer (CROSS): long-term results of a randomised controlled trial. Lancet Oncol. 2015;16:1090-8.

6. van Hagen P, Hulshof MC, van Lanschot JJ, Steyerberg EW, van Berge Henegouwen Ml, Wijnhoven BP, et al. Preoperative chemoradiotherapy for esophageal or junctional cancer. N Engl J Med. 2012;366:2074-84.

7. Ahmed O, Ajani JA, Lee JH. Endoscopic management of esophageal cancer. World J Gastrointest Oncol. 2019;11:830-41.

8. Pech O, May A, Manner H, Behrens A, Pohl J, Weferling M, et al. Long-term efficacy and safety of endoscopic resection for patients with mucosal adenocarcinoma of the esophagus. Gastroenterology. 2014;146:652-60.

9. Sharma P, Shaheen NJ, Katzka D, Bergman J. AGA clinical practice update on endoscopic treatment of barrett's esophagus with dysplasia and/or early cancer: expert review. Gastroenterology. 2020;158:760-9.

10. Cancer Genome Atlas Research Network. Comprehensive molecular characterization of gastric adenocarcinoma. Nature. 2014;513:202-9.

11. Cancer Genome Atlas Research Network et al. Integrated genomic characterization of oesophageal carcinoma. Nature. 2017;541:169-75.

12. Lordick F, Janjigian YY. Clinical impact of tumour biology in the management of gastroesophageal cancer. Nat Rev Clin Oncol. 2016;13:348-60.

13. Quail DF, Joyce JA. Microenvironmental regulation of tumor progression and metastasis. Nat Med. 2013;19:1423-37.

14. Biere SS, van Berge Henegouwen MI, Maas KW, Bonavina L, Rosman C, Garcia JR, et al. Minimally invasive versus open oesophagectomy for patients with oesophageal cancer: a multicentre, open-label, randomised controlled trial. Lancet. 2012;379:1887-92.
15. Mariette C, Markar SR, Dabakuyo-Yonli TS, Meunier B, Pezet D, Collet D, et al. Hybrid minimally invasive esophagectomy for esophageal cancer. New Engl J Med. 2019;380:152-62.

16. van der Sluis PC, van der Horst S, May AM, Schippers C, Brosens LAA, Joore HCA, et al. Robot-assisted minimally invasive thoracolaparoscopic esophagectomy versus open transthoracic esophagectomy for resectable esophageal cancer: a randomized controlled trial. Ann Surg. 2019;269:621-30.

17. Jayaprakasam VS, Yeh R, Ku GY, Petkovska I, Fuqua JL 3rd, Gollub M, et al. Role of imaging in esophageal cancer management in 2020: update for radiologists. AJR. 2020;215:1072-84.

18. Nicholas O, Prosser S, Mortensen HR, Radhakrishna G, Hawkins MA, Gwynne SH. The promise of proton beam therapy for oesophageal cancer: a systematic review of dosimetric and clinical outcomes. Clin Oncol (R Coll Radiol). 2021; S0936-6555(21)00152-7; e-pub ahead of print.

19. Graham D, Sever N, Magee C, Waddingham W, Banks M, Sweis R, et al. Risk of lymph node metastases in patients with $\mathrm{T} 1 \mathrm{~b}$ oesophageal adenocarcinoma: A retrospective single centre experience. World J Gastroenterol. 2018;24:4698-707.

20. Kolb JM, Wani S. Endoscopic eradication therapy for Barrett's oesophagus: state of the art. Curr Opin Gastroenterol. 2020;36:351-8.

21. Bergeron EJ, Lin J, Chang AC, Orringer MB, Reddy RM. Endoscopic ultrasound is inadequate to determine which $\mathrm{T} 1 / \mathrm{T} 2$ esophageal tumors are candidates for endoluminal therapies. J Thorac Cardiovasc Surg. 2014;147:765-71.

22. Participants in the Paris Workshop. The Paris endoscopic classification of superficial neoplastic lesions: esophagus, stomach, and colon: November 30 to December 1, 2002. Gastrointest Endosc. 2003; 58(6 Suppl):S3-43.

23. Ishihara R, Goda K, Oyama T. Endoscopic diagnosis and treatment of esophageal adenocarcinoma: introduction of Japan Esophageal Society classification of Barrett's esophagus. J Gastroenterol. 2019;54:1-9.

24. Ishihara R, Arima M, lizuka T, Oyama T, Katada C, Kato M, et al. Endoscopic submucosal dissection/endoscopic mucosal resection guidelines for esophageal cancer. Dig Endosc. 2020;32:452-93.

25. Oyama $T$, Inoue $H$, Arima $M$, Momma K, Omori $T$, Ishihara $R$, et al. Prediction of the invasion depth of superficial squamous cell carcinoma based on microvessel morphology: magnifying endoscopic classification of the Japan Esophageal Society. Esophagus. 2017;14:105-12.

26. Ueda T, Dohi O, Naito Y, Yoshida T, Azuma Y, Ishida T, et al. Diagnostic performance of magnifying blue laser imaging versus magnifying narrow-band imaging for identifying the depth of invasion of superficial esophageal squamous cell carcinoma. Dis Esophagus. 2020. https://doi.org/10.1093/dote/doaa078.

27. Kitagawa $\mathrm{Y}$, Uno T, Oyama T, Kato K, Kato H, Kawakubo H, et al. Esophageal cancer practice guidelines 2017 edited by the Japan esophageal society: part 2. Esophagus. 2019;16:25-43.

28. Kitagawa $\mathrm{Y}$, Uno T, Oyama $T$, Kato $\mathrm{K}$, Kato $\mathrm{H}$, Kawakubo $\mathrm{H}$, et al. Esophageal cancer practice guidelines 2017 edited by the Japan Esophageal Society: part 1. Esophagus. 2019;16:1-24.

29. Minashi K, Nihei K, Mizusawa J, Takizawa K, Yano T, Ezoe Y, et al. Efficacy of endoscopic resection and selective chemoradiotherapy for stage $i$ esophageal squamous cell carcinoma. Gastroenterology. 2019;157:382-90.

30. Cunningham D, Allum WH, Stenning SP, Thompson JN, Van de Velde CJ, Nicolson $\mathrm{M}$, et al. Perioperative chemotherapy versus surgery alone for resectable gastroesophageal cancer. New Engl J Med. 2006;355:11-20.

31. Ychou M, Boige V, Pignon JP, Conroy T, Bouche O, Lebreton G, et al. Perioperative chemotherapy compared with surgery alone for resectable gastroesophageal adenocarcinoma: an FNCLCC and FFCD multicenter phase III trial. J Clin Oncol. 2011;29:1715-21.

32. Gwynne S, Falk S, Gollins S, Wills L, Bateman A, Cummins S, et al. Oesophageal chemoradiotherapy in the UK-current practice and future directions. Clin Oncol. 2013;25:368-77.

33. Alderson D, Cunningham D, Nankivell M, Blazeby JM, Griffin SM, Crellin A, et al. Neoadjuvant cisplatin and fluorouracil versus epirubicin, cisplatin, and capecitabine followed by resection in patients with oesophageal adenocarcinoma (UK MRC OE05): an open-label, randomised phase 3 trial. Lancet Oncol. 2017;18:1249-60.

34. Glatz T, Verst R, Kuvendjiska J, Bronsert P, Becker H, Hoeppner J, et al. Pattern of recurrence and patient survival after perioperative chemotherapy with 5FU, leucovorin, oxaliplatin and docetaxel (FLOT) for locally advanced esophagogastric adenocarcinoma in patients treated outside clinical trials. J Clin Med. 2020;9:2654.

35. Burmeister BH, Thomas JM, Burmeister EA, Walpole ET, Harvey JA, Thomson DB, et al. Is concurrent radiation therapy required in patients receiving preoperative chemotherapy for adenocarcinoma of the oesophagus? A randomised phase II trial. Eur J Cancer. 2011;47:354-60.

36. Klevebro F, Alexandersson von Dobeln G, Wang N, Johnsen G, Jacobsen AB, Friesland $\mathrm{S}$, et al. A randomized clinical trial of neoadjuvant chemotherapy versus neoadjuvant chemoradiotherapy for cancer of the oesophagus or gastrooesophageal junction. Ann Oncol. 2016;27:660-7. 
37. Stahl M, Walz MK, Stuschke M, Lehmann N, Meyer HJ, Riera-Knorrenschild J, et al. Phase III comparison of preoperative chemotherapy compared with chemoradiotherapy in patients with locally advanced adenocarcinoma of the esophagogastric junction. J Clin Oncol. 2009;27:851-6.

38. Hoeppner J, Lordick F, Brunner T, Glatz T, Bronsert P, Rothling N, et al. ESOPEC: prospective randomized controlled multicenter phase III trial comparing perioperative chemotherapy (FLOT protocol) to neoadjuvant chemoradiation (CROSS protocol) in patients with adenocarcinoma of the esophagus (NCT02509286). BMC Cancer. 2016;16:503.

39. Reynolds JV, Preston SR, O'Neill B, Baeksgaard L, Griffin SM, Mariette C, et al. ICORG 10-14: neoadjuvant trial in adenocarcinoma of the oesophagus and oesophagogastric junction international study (Neo-AEGIS). BMC Cancer. 2017;17:401.

40. Leong T, Smithers BM, Michael M, Gebski V, Boussioutas A, Miller D, et al. TOPGEAR: a randomised phase III trial of perioperative ECF chemotherapy versus preoperative chemoradiation plus perioperative ECF chemotherapy for resectable gastric cancer (an international, intergroup trial of the AGITG/TROG/ EORTC/NCIC CTG). BMC Cancer. 2015;15:532.

41. Lorenzen S, Biederstadt A, Ronellenfitsch U, Reissfelder C, Monig S, Wenz F, et al. RACE-trial: neoadjuvant radiochemotherapy versus chemotherapy for patients with locally advanced, potentially resectable adenocarcinoma of the gastroesophageal junction-a randomized phase III joint study of the AIO, ARO and DGAV. BMC Cancer. 2020;20:886

42. Mukherjee S, Hurt CN, Gwynne S, Sebag-Montefiore D, Radhakrishna G, Gollins $S$, et al. NEOSCOPE: a randomised phase II study of induction chemotherapy followed by oxaliplatin/capecitabine or carboplatin/paclitaxel based preoperative chemoradiation for resectable oesophageal adenocarcinoma. Eur J Cancer. 2017;74:38-46.

43. Messager M, Mirabel X, Tresch E, Paumier A, Vendrely V, Dahan L, et al. Preoperative chemoradiation with paclitaxel-carboplatin or with fluorouraciloxaliplatin-folinic acid (FOLFOX) for resectable esophageal and junctional cancer: the PROTECT-1402, randomized phase 2 trial. BMC Cancer. 2016;16:318.

44. Goodman KA, Niedzwiecki D, Hall N, Bekaii-Saab TS, Ye X, Meyers MO, et al. Initial results of CALGB 80803 (alliance): a randomized phase II trial of PET scandirected combined modality therapy for esophageal cancer. J Clin Oncol. 2017;35:1-1.

45. Lordick F, Ott K, Krause BJ, Weber WA, Becker K, Stein HJ, et al. PET to assess early metabolic response and to guide treatment of adenocarcinoma of the oesophagogastric junction: the MUNICON phase II trial. Lancet Oncol. 2007;8:797-805.

46. Ott K, Weber WA, Lordick F, Becker K, Busch R, Herrmann K, et al. Metabolic imaging predicts response, survival, and recurrence in adenocarcinomas of the esophagogastric junction. J Clin Oncol. 2006;24:4692-8.

47. zum Buschenfelde CM, Herrmann K, Schuster T, Geinitz H, Langer R, Becker K, et al. (18)F-FDG PET-guided salvage neoadjuvant radiochemotherapy of adenocarcinoma of the esophagogastric junction: the MUNICON II trial. J Nucl Med. 2011;52:1189-96.

48. Sheil F, Donohoe $C L$, King $S$, O'Toole $D$, Cunningham M, Cuffe $S$, et al. Outcomes for esophageal squamous cell carcinoma treated with curative intent in a western cohort: should multimodal therapy be the gold standard? World J Surg. 2018;42:1485-95.

49. Yang $H$, Liu $H$, Chen $Y$, Zhu C, Fang W, Yu Z, et al. Neoadjuvant chemoradiotherapy followed by surgery versus surgery alone for locally advanced squamous cell carcinoma of the esophagus (NEOCRTEC5010): a phase III multicenter, randomized, open-label clinical trial. J Clin Oncol. 2018;36:2796-803.

50. Hara H, Tahara M, Daiko H, Kato K, Igaki H, Kadowaki S, et al. Phase II feasibility study of preoperative chemotherapy with docetaxel, cisplatin, and fluorouracil for esophageal squamous cell carcinoma. Cancer Sci. 2013;104:1455-60.

51. Koyanagi K, Kanamori K, Ninomiya Y, Yatabe K, Higuchi T, Yamamoto M, et al. Progress in multimodal treatment for advanced esophageal squamous cell carcinoma: results of multi-institutional trials conducted in Japan. Cancers. 2020;13:51.

52. Nakamura K, Kato K, Igaki H, Ito Y, Mizusawa J, Ando N, et al. Three-arm phase III trial comparing cisplatin plus 5-FU (CF) versus docetaxel, cisplatin plus 5-FU (DCF) versus radiotherapy with CF (CF-RT) as preoperative therapy for locally advanced esophageal cancer (JCOG1109, NExT study). Jpn J Clin Oncol. 2013;43:752-5.

53. Vellayappan BA, Soon YY, Ku GY, Leong CN, Lu JJ, Tey JC. Chemoradiotherapy versus chemoradiotherapy plus surgery for esophageal cancer. Cochrane Database Syst Rev. 2017;8:CD010511.

54. Stahl M, Stuschke M, Lehmann N, Meyer HJ, Walz MK, Seeber S, et al. Chemoradiation with and without surgery in patients with locally advanced squamous cell carcinoma of the esophagus. J Clin Oncol. 2005;23:2310-7.

55. Bedenne L, Michel P, Bouche O, Milan C, Mariette C, Conroy T, et al. Chemoradiation followed by surgery compared with chemoradiation alone in squamous cancer of the esophagus: FFCD 9102. J Clin Oncol. 2007;25:1160-8.

56. Kamarajah SK, Phillips AW, Hanna GB, Low D, Markar SR. Definitive chemoradiotherapy compared to neoadjuvant chemoradiotherapy with esophagectomy for locoregional esophageal cancer: national population-based cohort study. Ann Surg. 2020. https://doi.org/10.1097/SLA.0000000000003941.

57. Ito $Y$, Takeuchi H, Ogawa G, Kato K, Onozawa M, Minashi K, et al. A single-arm confirmatory study of definitive chemoradiotherapy (dCRT) including salvage treatment in patients (pts) with clinical (c) stage II/III esophageal carcinoma (EC) (JCOG0909). J Clin Oncol. 2018;36:4051.

58. Cooper JS, Guo MD, Herskovic A, Macdonald JS, Martenson JA Jr., Al-Sarraf M, et al. Chemoradiotherapy of locally advanced esophageal cancer: long-term follow-up of a prospective randomized trial (RTOG 85-01). Radiation Therapy Oncology Group. J Am Med Assoc. 1999;281:1623-7.

59. Ising MS, Marino K, Trivedi JR, Rojan AA, Dunlap NE, van Berkel V, et al. Influence of neoadjuvant radiation dose on patients undergoing esophagectomy and survival in locally advanced esophageal cancer. J Gastrointest Surg. 2019;23:670-8.

60. Boustani J, Rivin Del Campo E, Blanc J, Peiffert D, Benezery K, Pereira R, et al. Quality assurance of dose-escalated radiation therapy in a randomized trial for locally advanced oesophageal cancer. Int J Radiat Oncol Biol Phys. 2019;105:329-37.

61. Hulshof MC, Geijsen D, Rozema T, Oppedijk V, Buijsen J, Neelis KJ, et al. A randomized controlled phase III multicenter study on dose escalation in definitive chemoradiation for patients with locally advanced esophageal cancer: ARTDECO study. J Clin Oncol. 2020;38:281.

62. Warren S, Partridge M, Carrington R, Hurt C, Crosby T, Hawkins MA. Radiobiological determination of dose escalation and normal tissue toxicity in definitive chemoradiation therapy for esophageal cancer. Int J Radiat Oncol Biol Phys. 2014;90:423-9.

63. Blazeby JM, Strong S, Donovan JL, Wilson C, Hollingworth W, Crosby T, et al. Feasibility RCT of definitive chemoradiotherapy or chemotherapy and surgery for oesophageal squamous cell cancer. Br J Cancer. 2014;111:234-40.

64. D'Journo XB, Michelet P, Dahan L, Doddoli C, Seitz JF, Giudicelli R, et al. Indications and outcome of salvage surgery for oesophageal cancer. Eur J Cardiothorac Surg. 2008;33:1117-23.

65. Gergelis KR, Jethwa KR, Tryggestad EJ, Ashman JB, Haddock MG, Hallemeier CL. Proton beam radiotherapy for esophagus cancer: state of the art. J Thorac Dis. 2020;12:7002-10.

66. Wahed S, Chmelo J, Navidi M, Hayes N, Phillips AW, Immanuel A. Delivering esophago-gastric cancer care during the COVID-19 pandemic in the United Kingdom: a surgical perspective. Dis Esophagus. 2020;33:doaa091.

67. Noordman BJ, Spaander MCW, Valkema R, Wijnhoven BPL, van Berge Henegouwen MI, Shapiro J, et al. Detection of residual disease after neoadjuvant chemoradiotherapy for oesophageal cancer (preSANO): a prospective multicentre, diagnostic cohort study. Lancet Oncol. 2018;19:965-74.

68. Van Der Wilk B, Eyck B, Hofstetter W, Ajani J, Piessen G, Castoro C, et al. Chemoradiotherapy followed by active surveillance versus surgery for esophageal cancer: a systematic review and individual patient data meta-analysis. Dis Eso phagus. 2020:33;S1:doaa087.50

69. Cheedella NK, Suzuki A, Xiao L, Hofstetter WL, Maru DM, Taketa T, et al. Association between clinical complete response and pathological complete response after preoperative chemoradiation in patients with gastroesophageal cancer: analysis in a large cohort. Ann Oncol. 2013;24:1262-6.

70. Triantafyllou T, Wijnhoven B. Multidisciplinary treatment of esophageal cancer: the role of active surveillance after neoadjuvant chemoradiation. Ann Gastroenterol Surg. 2020;4:352-9.

71. Heneghan HM, Donohoe C, Elliot J, Ahmed Z, Malik V, Ravi N, et al. Can CT-PET and endoscopic assessment post-neoadjuvant chemoradiotherapy predict residual disease in esophageal cancer? Ann Surg. 2016;264:831-8.

72. Noordman BJ, Wijnhoven BPL, Lagarde SM, Boonstra JJ, Coene $P$, Dekker JWT, et al. Neoadjuvant chemoradiotherapy plus surgery versus active surveillance for oesophageal cancer: a stepped-wedge cluster randomised trial. BMC Cancer. 2018;18:142.

73. CHU D. Comparison of systematic surgery versus surveillance and rescue surgery in operable oesophageal cancer with a complete clinical response to radiochemotherapy (esostrate) NCT02551458. 2015. https://clinicaltrials.gov/ct2/ show/NCT02551458. Accessed 8 February 2021.

74. Zhang X, Eyck BM, Yang Y, Liu J, Chao YK, Hou MM, et al. Accuracy of detecting residual disease after neoadjuvant chemoradiotherapy for esophageal squamous cell carcinoma (preSINO trial): a prospective multicenter diagnostic cohort study. BMC Cancer. 2020;20:194.

75. Noordman BJ, de Bekker-Grob EW, Coene P, van der Harst E, Lagarde SM, Shapiro J, et al. Patients' preferences for treatment after neoadjuvant chemoradiotherapy for oesophageal cancer. Br J Surg. 2018;105:1630-8.

76. Karolinska UH. A study of chemoradiotherapy followed by planned surgery or by surveillance and surgery only when needed for cancer of the esophagus (NEEDS) NCT04460352. 2020. https://clinicaltrials.gov/ct2/show/NCT04460352. Accessed 20 March 2021.

77. Bang YJ, Van Cutsem E, Feyereislova A, Chung HC, Shen L, Sawaki A, et al. Trastuzumab in combination with chemotherapy versus chemotherapy alone for 
treatment of HER2-positive advanced gastric or gastro-oesophageal junction cancer (ToGA): a phase 3, open-label, randomised controlled trial. Lancet. 2010;376:687-97.

78. Fuchs CS, Tomasek J, Yong CJ, Dumitru F, Passalacqua R, Goswami C, et al. Ramucirumab monotherapy for previously treated advanced gastric or gastrooesophageal junction adenocarcinoma (REGARD): an international, randomised, multicentre, placebo-controlled, phase 3 trial. Lancet. 2014;383:31-9.

79. Hironaka S, Shimada Y, Sugimoto N, Komatsu Y, Nishina T, Yamaguchi K, et al. RAINBOW: a global, phase III, randomized, double-blind study of ramucirumab (RAM) plus paclitaxel (PTX) versus placebo (PL) plus PTX in the treatment of metastatic gastroesophageal junction and gastric adenocarcinoma (mGC) following disease progression on first-line platinum- and fluoropyrimidinecontaining combination therapy-efficacy analysis in Japanese and Western patients. J Clin Oncol. 2014;32:4005.

80. Hofheinz RD, Haag GM, Ettrich TJ, Borchert K, Kretzschmar A, Teschendorf C, et al. Perioperative trastuzumab and pertuzumab in combination with FLOT versus FLOT alone for HER2-positive resectable esophagogastric adenocarcinoma: final results of the PETRARCA multicenter randomized phase II trial of the AIO. J Clin Oncol. 2020;38:4502.

81. Samstein RM, Lee CH, Shoushtari AN, Hellmann MD, Shen R, Janjigian YY, et al. Tumor mutational load predicts survival after immunotherapy across multiple cancer types. Nat Genet. 2019;51:202-6.

82. Kelly RJ. The emerging role of immunotherapy for esophageal cancer. Curr Opin Gastroenterol. 2019;35:337-43.

83. Fuchs CS, Doi T, Jang RW, Muro K, Satoh T, Machado M, et al. Safety and efficacy of pembrolizumab monotherapy in patients with previously treated advanced gastric and gastroesophageal junction cancer: phase 2 clinical KEYNOTE-059 trial. JAMA Oncol. 2018;4:e180013.

84. Kang YK, Boku N, Satoh T, Ryu MH, Chao Y, Kato K, et al. Nivolumab in patients with advanced gastric or gastro-oesophageal junction cancer refractory to, or intolerant of, at least two previous chemotherapy regimens (ONO-4538-12, ATTRACTION-2): a randomised, double-blind, placebo-controlled, phase 3 trial. Lancet. 2017;390:2461-71.

85. Moehler M, Shitara K, Garrido M, Salman P, Shen L, Wyrwicz L, et al. LBA6_PR Nivolumab (nivo) plus chemotherapy (chemo) versus chemo as first-line (1L) treatment for advanced gastric cancer/gastroesophageal junction cancer (GC/ GEJC)/esophageal adenocarcinoma (EAC): First results of the CheckMate 649 study. Ann Oncol. 2020;31(Suppl 4):S1191.

86. Kato K, Cho BC, Takahashi M, Okada M, Lin CY, Chin K, et al. Nivolumab versus chemotherapy in patients with advanced oesophageal squamous cell carcinoma refractory or intolerant to previous chemotherapy (ATTRACTION-3): a multicentre, randomised, open-label, phase 3 trial. Lancet Oncol. 2019:20:1506-17.

87. Janjigian YY, Maron SB, Chatila WK, Millang B, Chavan SS, Alterman C, et al. Firstline pembrolizumab and trastuzumab in HER2-positive oesophageal, gastric, or gastro-oesophageal junction cancer: an open-label, single-arm, phase 2 trial. Lancet Oncol. 2020;21:821-31.

88. Kelly RJ, Ajani AJ, Kuzdzal J, Zander T, Van Cutsem E, Piessen G, et al. Adjuvant nivolumab in resected esophageal or gastroesophageal junction cancer. $\mathrm{N}$ Engl J Med. 2021;384:1191-203.

89. Donlon NE, Power R, Hayes C, Reynolds JV, Lysaght J. Radiotherapy, immunotherapy, and the tumour microenvironment: turning an immunosuppressive milieu into a therapeutic opportunity. Cancer Lett. 2021;502:84-96.

90. Ngwa W, Irabor OC, Schoenfeld JD, Hesser J, Demaria S, Formenti SC. Using immunotherapy to boost the abscopal effect. Nat Rev Cancer. 2018;18:313-22.

91. Rosenbaum MW, Gonzalez RS. Targeted therapy for upper gastrointestinal tract cancer: current and future prospects. Histopathology. 2021;78:148-61.

92. Cunningham D, Stenning SP, Smyth EC, Okines AF, Allum WH, Rowley S, et al. Peri-operative chemotherapy with or without bevacizumab in operable oesophagogastric adenocarcinoma (UK Medical Research Council STO3): primary analysis results of a multicentre, open-label, randomised phase 2-3 trial. Lancet Oncol. 2017;18:357-70.

93. Park SY, Hong MH, Kim HR, Lee CG, Cho JH, Cho BC, et al. The feasibility and safety of radical esophagectomy in patients receiving neoadjuvant chemoradiotherapy with pembrolizumab for esophageal squamous cell carcinoma. J Thorac Dis. 2020;12:6426-34.

94. Sihag S, Ku GY, Tan KS, Nussenzweig S, Wu A, Janjigian YY, et al. Safety and feasibility of esophagectomy following combined immunotherapy and chemoradiotherapy for esophageal cancer. J Thorac Cardiovasc Surg. 2020. https:// doi.org/10.1016/j.jtcvs.2020.11.106.

95. Holscher AH, Law S. Esophagogastric junction adenocarcinomas: individualization of resection with special considerations for Siewert type II, and Nishi types $E G, E=G$ and GE cancers. Gastric Cancer. 2020;23:3-9.

96. Hulscher JB, van Sandick JW, de Boer AG, Wijnhoven BP, Tijssen JG, Fockens P, et al. Extended transthoracic resection compared with limited transhiatal resection for adenocarcinoma of the esophagus. New Engl J Med. 2002;347:1662-9.
97. Gottlieb-Vedi E, Kauppila JH, Malietzis G, Nilsson M, Markar SR, Lagergren J. Long-term survival in esophageal cancer after minimally invasive compared to open esophagectomy: a systematic review and meta-analysis. Ann Surg. 2019;270:1005-17.

98. Cuschieri A, Shimi S, Banting S. Endoscopic oesophagectomy through a right thoracoscopic approach. J R Coll Surg Edinb. 1992;37:7-11.

99. Luketich JD, Pennathur A, Awais O, Levy RM, Keeley S, Shende M, et al. Outcomes after minimally invasive esophagectomy: review of over 1000 patients. Ann Surg. 2012;256:95-103.

100. Straatman J, van der Wielen N, Cuesta MA, Daams F, Roig Garcia J, Bonavina L, et al. Minimally invasive versus open esophageal resection: three-year follow-up of the previously reported randomized controlled trial: the TIME trial. Ann Surg. 2017;266:232-6.

101. Markar SR, Ni M, Gisbertz SS, van der Werf L, Straatman J, van der Peet D, et al. Implementation of minimally invasive esophagectomy from a randomized controlled trial setting to national practice. J Clin Oncol. 2020;38:2130-9.

102. Reynolds JV, Donlon N, Elliott JA, Donohoe C, Ravi N, Kuppusamy MK, et al. Comparison of esophagectomy outcomes between a national center, a national audit collaborative, and an international database using the Esophageal Complications Consensus Group (ECCG) standardized definitions. Dis Esophagus. 2021;34:doaa060.

103. Wijnhoven BPL, Lagarde SM. Minimally invasive esophagectomy: time to reflect on contemporary outcomes. J Clin Oncol. 2021;39:90-1.

104. Brierley RC, Gaunt D, Metcalfe C, Blazeby JM, Blencowe NS, Jepson M, et al. Laparoscopically assisted versus open oesophagectomy for patients with oesophageal cancer-the randomised oesophagectomy: minimally invasive or open (ROMIO) study: protocol for a randomised controlled trial (RCT). BMJ Open. 2019;9: e030907.

105. Kernstine KH, DeArmond DT, Karimi M, Van Natta TL, Campos JH, Yoder MR, et al. The robotic, 2-stage, 3-field esophagolymphadenectomy. J Thorac Cardiovasc Surg. 2004;127:1847-9.

106. de Groot EM, van der Horst S, Kingma BF, Goense L, van der Sluis PC, Ruurda JP, et al. Robot-assisted minimally invasive thoracolaparoscopic esophagectomy versus open esophagectomy: long-term follow-up of a randomized clinical trial. Dis Esophagus. 2020;33(Suppl 2):doaa079.

107. Kingma BF, Grimminger PP, van der Sluis PC, van Det MJ, Kouwenhoven EA, Chao YK, et al. Worldwide techniques and outcomes in robot-assisted minimally invasive esophagectomy (RAMIE): results from the multicenter international registry. Ann Surg. 2020. https://doi.org/10.1097/SLA.0000000000004550.

108. Evans RPT, Singh P, Nepogodiev D, Bundred J, Kamarajah S, Jefferies B, et al. Study protocol for a multicenter prospective cohort study on esophagogastric anastomoses and anastomotic leak (the Oesophago-Gastric Anastomosis Audit/ OGAA). Dis Esophagus. 2020;33:1-15.

109. Pennathur A, Godfrey TE, Luketich JD. The molecular biologic basis of esophageal and gastric cancers. Surg Clin North Am. 2019;99:403-18.

110. Pennathur A, Xi L, Litle VR, Gooding WE, Krasinskas A, Landreneau RJ, et al. Gene expression profiles in esophageal adenocarcinoma predict survival after resection. J Thorac Cardiovasc Surg. 2013;145:505-12.

111. Boniface C, Deig C, Halsey C, Kelley T, Heskett MB, Thomas CR Jr., et al. The feasibility of patient-specific circulating tumor DNA monitoring throughout multi-modality therapy for locally advanced esophageal and rectal cancer: a potential biomarker for early detection of subclinical disease. Diagnostics. 2021;11:73.

112. Iwaya T, Endo F, Takahashi F, Tokino T, Sasaki Y, Nishizuka SS. Frequent tumor burden monitoring of esophageal squamous cell carcinoma with circulating tumor DNA using individually designed digital polymerase chain reaction. Gastroenterology. 2021;160:463-5.

\section{AUTHOR CONTRIBUTIONS}

Manuscript concept: JCB and JVR; writing/editing: JCB, CLD, ML and JVR; revising manuscript: JCB, CLD, ML and JVR. All authors have approved the final version of this manuscript.

\section{FUNDING}

Open Access funding provided by the IReL Consortium.

\section{COMPETING INTERESTS}

The authors declare no competing interests.

\section{ETHICS APPROVAL AND CONSENT TO PARTICIPATE}

Not applicable. 
CONSENT TO PUBLISH

Not applicable.

\section{ADDITIONAL INFORMATION}

Correspondence and requests for materials should be addressed to John. V. Reynolds.

Reprints and permission information is available at http://www.nature.com/ reprints

Publisher's note Springer Nature remains neutral with regard to jurisdictional claims in published maps and institutional affiliations.
Open Access This article is licensed under a Creative Commons Cc) Attribution 4.0 International License, which permits use, sharing,
adaptation, distribution and reproduction in any medium or format, as long as you give appropriate credit to the original author(s) and the source, provide a link to the Creative Commons license, and indicate if changes were made. The images or other third party material in this article are included in the article's Creative Commons license, unless indicated otherwise in a credit line to the material. If material is not included in the article's Creative Commons license and your intended use is not permitted by statutory regulation or exceeds the permitted use, you will need to obtain permission directly from the copyright holder. To view a copy of this license, visit http://creativecommons. org/licenses/by/4.0/.

(c) The Author(s) 2021 